\title{
Side Rail Device
}

National Cancer Institute

\section{Source}

National Cancer Institute. Side Rail Device. NCI Thesaurus. Code C50301.

A supportive or protective rail attached to the side of something. 